\title{
LACTB-mediated tumour suppression by increased mitochondrial lipid metabolism
}

\author{
Danilo Cucchi ${ }^{1,2}$ and Claudio Mauro, \\ Cell Death and Differentiation (2017) 24, 1137-1139; doi:10.1038/cdd.2017.60; published online 5 May 2017
}

Metabolism has long been known to be a driver of cancer. In the 1920s, Otto Warburg formulated the hypothesis that virtually all cancer cells become addicted to glucose and glucose metabolism with the production of large amounts of lactate, even in conditions of oxygen availability. This metabolic reprogramming, coined as 'Warburg effect'or 'aerobic glycolysis', indeed favours cell transformation and tumour growth. ${ }^{1}$ Yet aerobic glycolysis does not explain many of the metabolic changes that occur in cancer, nor it is just a passive adaptation to damaged mitochondria, which in fact are often intact and functional in cancers. ${ }^{2}$ We are only starting to understand the biochemical and molecular basis of the metabolic requirements of cancers, but altered metabolism is now viewed as a hallmark of cancer. ${ }^{2,3}$

Much focus has been devoted to oncogenic mechanisms. Classical oncogenes are capable of driving anabolic growth via direct control of metabolic genes. Examples are KRAS leading to the upregulation of the glucose transporter GLUT1, or Myc causing increased expression of LDHA and GLS, involved in lactate generation and mitochondrial glutamine metabolism, respectively. Some metabolic enzymes have been shown to act directly as oncogenes such as PKM2 and PFKFB3 via means of alternative splicing mechanisms as well as hexokinase II, fatty acid synthase and others via increased copy number. Last but not the least, a number of metabolites present oncogenic features, such as $2 \mathrm{HG}$, fumarate, succinate and lactate. ${ }^{2,4}$

Some work also showed the interconnections that exist between tumour suppression and metabolism. One key example is p53 whose loss in tumours leads to enhanced glycolysis and biosynthesis supporting tumour growth. ${ }^{5}$ Yet again some metabolic enzymes acting within the TCA cycle in the mitochondria, IDH, $\mathrm{FH}$ and $\mathrm{SDH}$ have been shown to act as tumour suppressors with their loss causing accumulation of substrates acting as onco-metabolites. ${ }^{6}$

Hence, do mitochondia carry a broader tumour-suppressing potential? A new study from Keckesova et al. ${ }^{7}$ addressed this question starting from the very common observation that some tissues almost never undergo cancer transformation. This is the case of heart, skeletal muscle or neurons, and in contrast to tissues such as breast, lungs or colon, where the incidence of cancer is high. Characteristically, cells in cancer-resistant tissues are non-proliferative, terminally differentiated and oxidative, unlike cancer cells that are proliferative, undifferentiated and highly glycolytic. This led the authors to hypothesise that the analysis of the gene expression profiles of cancer-resistant tissues may enable the identification of novel tumour suppressors. Such approach is a one-of-a-kind, in sharp contrast to the majority of existing studies in which the genes and mechanisms involved with cancer transformation have been investigated in tissues where cancer often develops.

The authors found that the mitochondrial protein LACTB may act as a tumour suppressor via its action on mitochondrial lipid metabolism, ultimately leading to increased differentiation and reduced proliferation of breast cancer cells (Figure 1). A microarray analysis was conducted to identify genes upregulated in differentiated post-mitotic human and murine muscle cells as compared to their actively cycling progenitors. On the basis of expression profiles, five genes were chosen for functional validation. LACTB overexpression had the greatest impact on decreasing the rate of proliferation of breast cancer cell lines with only minimal effect on the proliferation of non-tumourigenic cell lines. LACTB protein levels were downregulated in 15 out of 18 breast cancer cell lines as compared to primary mammary cells. Even more importantly, they were downregulated in over a third breast cancer tissues out of 714 clinical samples as compared to the 120 normal human breast tissues analysed. Exogenous expression of LACTB in already-formed tumours caused tumour regression, with instances of complete disappearance of the tumour mass. In contrast, LACTB silencing in non-transformed cells was not sufficient per se to cause transformation, but when combined to the expression of the oncogenes RAS or MYC, it caused the formation of tumours in vivo, unlike the expression of the oncogenes alone. Because the loss of a tumour suppressor is in most cases not sufficient but requires the concomitant expression of an oncogene to cause tranformation, this set of experiments is perhaps the strongest proof that LACTB is a bona fide new tumour suppressor.

Mechanistically, LACTB expression in breast cancer cells induced the expression of the markers of epithelial differentiation EPCAM and CD24, while at the same time reducing the cancer stem-cell markers CD44 and ZEB1 (Figure 1). But

\footnotetext{
${ }^{1}$ William Harvey Research Institute, Barts and The London School of Medicine and Dentistry, Queen Mary University of London, London, UK and ${ }^{2}$ Istituto Pasteur, Fondazione Cenci Bolognetti, Rome, Italy

${ }^{*}$ Corresponding author: C Mauro, William Harvey Research Institute, Barts and The London School of Medicine and Dentistry, Queen Mary University of London, Charterhouse Square, London EC1M 6BQ, UK. E-mail: c.mauro@qmul.ac.uk
} 


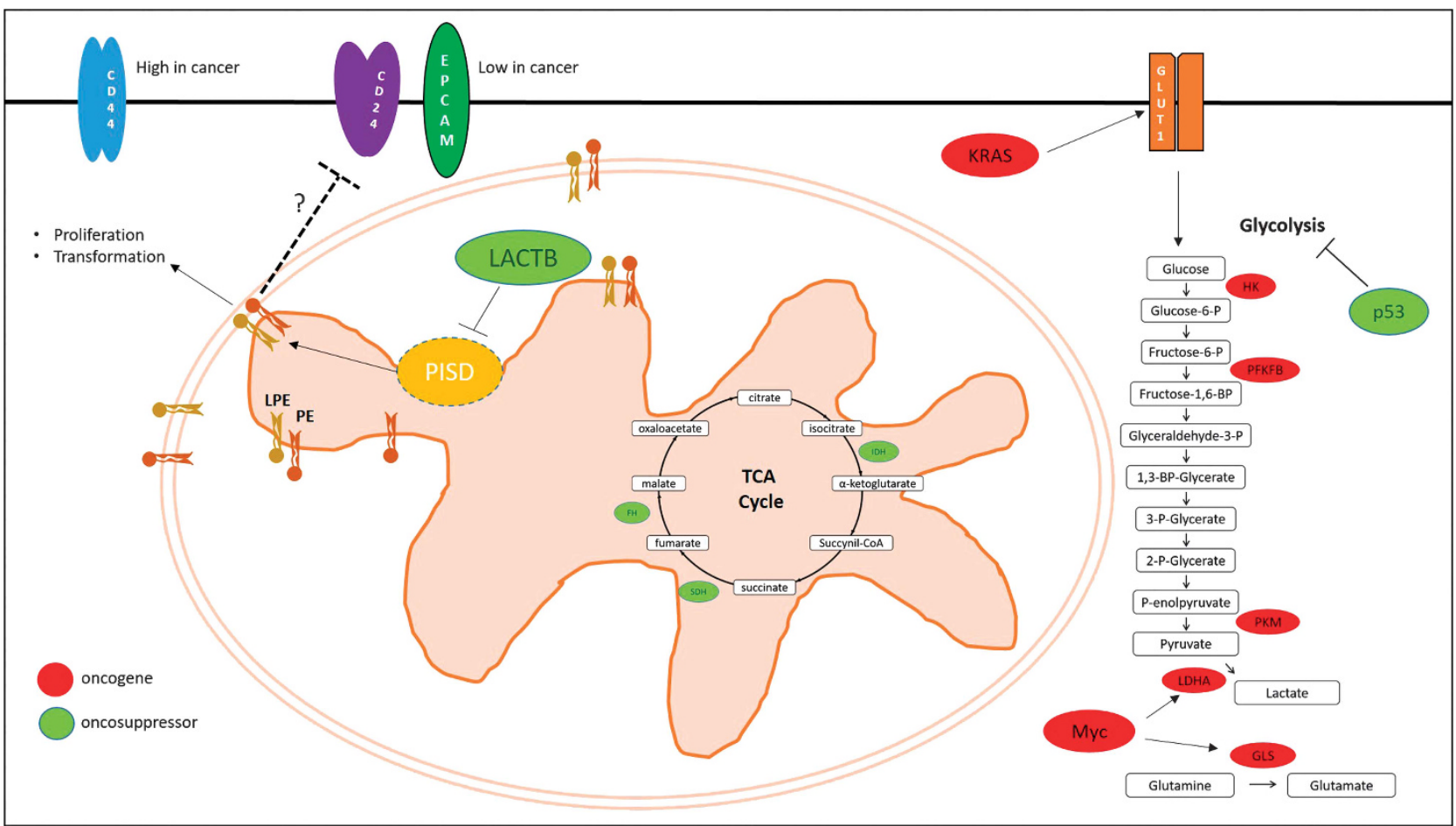

Figure 1 Interplay between oncogenes/oncosuppressors and cellular metabolism. Classical oncogenes can act through the regulation of metabolism, such as KRAS upregulating GLUT1, and Myc increasing the activity of LDHA and GLS; enzymes of the glycolysis, such as HK, PFKFB and PKM, are able themselves to promote cancer proliferation. On the contrary, p53, the best-known oncosuppressor gene, is able to downregulate the activity of glycolysis, and enzymes of the TCA cycle, namely, IDH, SDH and $\mathrm{FH}$, have been found to have oncosuppressor-like activity. Keckesova et al. have identified a new oncosuppressor gene, LACTB. LACTB is a mitochondrial protease able to downregulate the activity of a decarboxylase, PISD, which is responsible for the formation of membrane lipids PEs and LPEs. The loss of LACTB in cancer results in increased quantity of PE and LPE in mitochondrial membranes, which leads to increased proliferation and upregulation of CD44, a marker of cancer stem cells. Restoring LACTB expression in cancer cells results in reduction of PISD activity and reduced accumulation of PEs and LPEs. These changes are associated to reduced proliferation and acquisition of a more differentiated status, as indicated by the upregulation of CD24 and EPCAM, markers of epithelial differentiation. FH, fumarase; GLS, glutaminase; HK, exokinase; IDH, isocitrate dehydrogenase; LACTB, beta-lactamase-like protein; LDHA, lactate dehydrogenase A; LPE, lysophosphatidylethanolamine; PE, phosphatidylethanolamine; PFKFB, phosphofructokinase; PISD, phosphatidylserine decarboxylase; PKM, pyruvate kinase; SDH, succinate dehydrogenase; TCA cycle, tricarboxylic acid cycle

whether this effect was in any way linked to the cellular function of LACTB was the next major question to answer. The authors went on to analyse several mitochondrial processes and found a substancial decrease in the quantities of the mitochondial lipids lysophosphatidylethanolamines (LPEs) and phosphatidylethanolamines (PEs) upon LACTB expression in breast cancer cells but not in non-transformed cells. Supplementation of LACTB-expressing breast cancer cells with LPE was able to restore proliferative capacity and even induce it further over parental cells, and at the same time reduce the levels of the differentiation marker CD24 as compared to the levels in non-transfected cancer cells (Figure 1). Downstream of LACTB, the mitochondrial enzyme phosphatidylserine decarboxilase (PISD), converting phosphatidylserine to PEs, was strongly downregulated at posttrascriptional level. Silencing of $P I S D$ recapitulated the effects of overexpression of LACTB, that is, reduced proliferation of breast cancer cells (Figure 1). The downregulation of PISD required the proteolytic activity of $L A C T B$, although a direct interaction between the two proteins was not observed. In line with the lipid profiles data, the levels of PISD were reduced upon LACTB expression in breast cancer cells but not in non-tumourigenic mammary cells, providing a potential explanation for the differential effect of LACTB on the proliferation of tumourigenic and non-tumourigenic cells.

The study goes a long way to identify a new tumour suppressor gene and its mechanism of action. A number of open questions, however, remains. How LPEs control specific cell differentiation programs downstream of LCATB-PSID axis is yet not clear. More broadly, how lipids direct differentiation is an open question in many research fields. In obesity, palmitate was recently shown to be able to induce a programme of $\mathrm{CD}^{+}{ }^{+}$T-cell differentiation towards an effector memory proinflammatory phenotype via engaging a PI3K-Akt pathway. ${ }^{8,9}$ Exactly what the proximal events are in the palmitate induction of this pathway and what is downstream leading to increased expression of CXCR3 and LFA1 remains to be defined. Another puzzling question is how the selectivity of LACTB-mediated effects is achieved in tumourigenic versus non-tumourigenic cells. This is particularly relevant to the reported downregulation of PSID and consequent decrease in the amounts of LPEs and PEs. It would be similarly important to understand whether LACTB loss correlates with any particular breast tumour and occurs in other cancers of the body. At the biochemical level, how LACTB induces 
dowregulation of PISD remains to be elucidated. The proteolytic activity of LACTB seems to be required; however, LACTB and PISD do not appear to interact directly. Therefore, other currently unknow factors may be involved in the process. In this context, it is puzzling the fact that the mutant LACTB $^{\mathrm{R} 469 \mathrm{~K}}$ expressed in MCF7-RAS breast cancer cells, although keeping its proteolytic activity intact, is not able to downregulate PISD and LPE/PE lipid species, and ultimately to inhibit proliferation.

Overall, the study uncovers a novel tumour suppressor, the protease LACTB acting via the control of mitochondrial lipid metabolism. Perhaps more importantly, it provides a platform to identify genes and mechanisms operating in cancer-resistant tissues that can become novel targets for anti-cancer therapies.

\section{Conflict of Interest}

The authors declare no conflict of interest.

1. Vander Heiden MG, Cantley LC, Thompson CB. Science 2009; 324: 1029-1033.

2. Ward PS, Thompson CB. Cancer Cell 2012; 21: 297-308.

3. Hanahan D, Weinberg RA. Cell 2011; 331: 1559-1564.

4. Haas R et al. Trends Biochem Sci 2016; 41: 460-471.

5. Berkers CR et al. Cell Metab 2013; 18: 617-633.

6. Laurenti G, Tennant DA. Biochem Soc Trans 2016; 44: 1111-1116.

7. Keckesova $Z$ et al. Nature 2017; 543: 681-686.

8. Mauro C et al. Cell Metab 2017; 25: 593-609.

9. Chapman NM, Chi H. Cell Metab 2017; 25: 490-492. 